\title{
OTIMIZAÇÃO DA ETAPA DE PURIFICAÇÃO DA CERA BRUTA UTILIZANDO A FILTRAÇÃO
}

\author{
J.G.ROCHA ${ }^{1}$, J.MISSAU ${ }^{1}$, G.L.DOTTO ${ }^{1}$, L.P.CERON ${ }^{2}$ e E.H.TANABE ${ }^{1}$ \\ ${ }^{1}$ Universidade Federal de Santa Maria, Departamento de Engenharia Química \\ E-mail para contato: degregorijuliana@gmail.com \\ ${ }^{2}$ Pontifícia Universidade Católica do Rio Grande do Sul, Faculdade de Engenharia, \\ Departamento de Engenharia Química
}

\begin{abstract}
RESUMO - A filtração da cera tem como objetivo reduzir o teor de inorgânicos, com o propósito de se ter um produto que atenda as exigências do mercado, isto é, uma cera com alto grau de pureza. Para isso, procedeu-se a filtração na qual variou-se a temperatura e pressão em faixas pré-determinadas estatisticamente para se obter uma maior quantidade de massa de cera filtrada. Diante disso, variou-se a temperatura de $140-170^{\circ} \mathrm{C}$ e a pressão de $1-4$ bar. Os resultados mostraram que a faixa de temperatura e pressão que se obteve maior massa de cera filtrada foi $170^{\circ} \mathrm{C}$ e 2,5 bar, respectivamente. O percentual médio da remoção de impurezas da cera foi de $99,6 \%$, garantindo a ótima qualidade do produto obtido.
\end{abstract}

\section{INTRODUÇÃO}

No cenário atual, as ceras possuem uma ampla utilização. As aplicações das ceras atingem diversos ramos, tais como: cosméticos, giz de cera, adesivos, tinturas, explosivos e diversos outros (Wiley-VCH 2011).

A obtenção desse material pode ser feita, entre outras maneiras, pela pirólise de resíduos plásticos (Miandad et al. 2016). Porém, a cera obtida por pirólise necessita ser purificada, afim de reduzir o teor de inorgânicos aderidos a sua estrutura.

Diante disso, a operação unitária de filtração foi utilizada para remoção da fração inorgânica. O produto de interesse é a cera filtrada. Ao tratar-se de um fluido viscoso, a adição de um auxiliar de filtração pode melhorar a eficiência da operação de filtração (Du et al. 2011). Neste projeto escolheu-se a perlita como auxiliar de filtração.

Logo, o presente trabalho tem como objetivo otimizar as condições de filtração para obtenção de uma maior quantidade de massa de cera filtrada. Na otimização da filtração utilizou-se o método de delineamento composto central rotacional (DCCR). Recomenda-se a escolha deste método ao dispor de duas variáveis independentes (Rodrigues \& Iemma 2014). As variáveis independentes foram temperatura e pressão. A variável dependente principal foi a quantidade de massa de cera filtrada. 


\section{MATERIAIS E MÉTODOS}

\subsection{Materiais}

A cera bruta foi obtida pela empresa Alkaest, Brasil. A perlita foi adquirida da empresa Imerys, Argentina. O meio filtrante foi obtido da empresa Renner Têxtil, Brasil. O óleo Mobiltherm 605 para aquecimento do cilindro encamisado foi adquirido da empresa Lubritec, Brasil.

\subsection{Caracterização do meio filtrante}

O meio filtrante é um novo têxtil de para amida, também conhecido como Kevlar, sem tratamento físico. O mesmo possui resistência térmica e mecânica. As características principais do meio filtrante constam na tabela 1.

Tabela 1 - Características do meio filtrante.

\begin{tabular}{|c|c|c|}
\hline Parâmetros & Valor médio & Método de análise \\
\hline Gramatura & $546\left(\mathrm{~g} / \mathrm{m}^{2}\right)$ & $\begin{array}{c}\text { NBR 12984:2009 - Não tecido - Determinação da } \\
\text { massa por unidade de área }\end{array}$ \\
\hline Espessura & $3,0(\mathrm{~mm})$ & $\begin{array}{c}\text { NBR 13371:2005 - Não tecido - Determinação da } \\
\text { espessura }\end{array}$ \\
\hline Permeabilidade & $235\left(\mathrm{~L} / \mathrm{min}_{\mathrm{dm}} \mathrm{dm}^{2}\right)$ & $\begin{array}{c}\text { NBR 13706:1996 - Não tecido - Determinação da } \\
\text { permeabilidade ao ar }\end{array}$ \\
\hline
\end{tabular}

\subsection{Ensaios de filtração}

Para os experimentos de filtração realizou-se um DCCR com 11 ensaios. Variou-se a temperatura de $140-170^{\circ} \mathrm{C}$. A pressão foi variada de 1-4 bar. Os valores máximos e mínimos, dos parâmetros, foram baseados em testes preliminares. $\mathrm{O}$ equipamento utilizado nos ensaios de filtração está apresentado na Figura 1.

No primeiro momento, a cera bruta foi quarteada para garantir a homogeneidade das amostras (Mullin 2001). Posteriormente, $3 \%$ em peso de perlita foi adicionado à cera quarteada (Purchas \& Sutherland 2002). A mistura foi aquecida até tornar-se líquida. O meio filtrante foi aprisionado em um aparato formado por duas flanges metálicas. Uma tela metálica foi posicionada na flange inferior, funcionando como suporte para o filtro. Acima do meio filtrante, depositou-se a pré-capa de $3 \mathrm{~mm}$ de perlita em pó (Purchas \& Sutherland 2002). Após montagem do meio filtrante, o aparato foi conectado ao fundo do cilindro encamisado. A mistura de cera e perlita foi alimentada no topo do cilindro. Ao alcançar o equilíbrio térmico do sistema, o cilindro foi fechado. Conectou-se então a tubulação de gás 
nitrogênio. A vazão de gás foi controlada por um manômetro. A pressão foi mantida constante ao longo do processo de filtração para cada ensaio. Contou-se o tempo de filtração, mantido em $10 \mathrm{~min}$, a partir da primeira gota de cera filtrada. O material filtrado depositou-se em um béquer. Após atingir temperatura ambiente, pesou-se a massa de cera filtrada e efetuou-se o teste de inorgânicos.

Figura 1 - Equipamento de filtração desenvolvido para purificação de ceras.

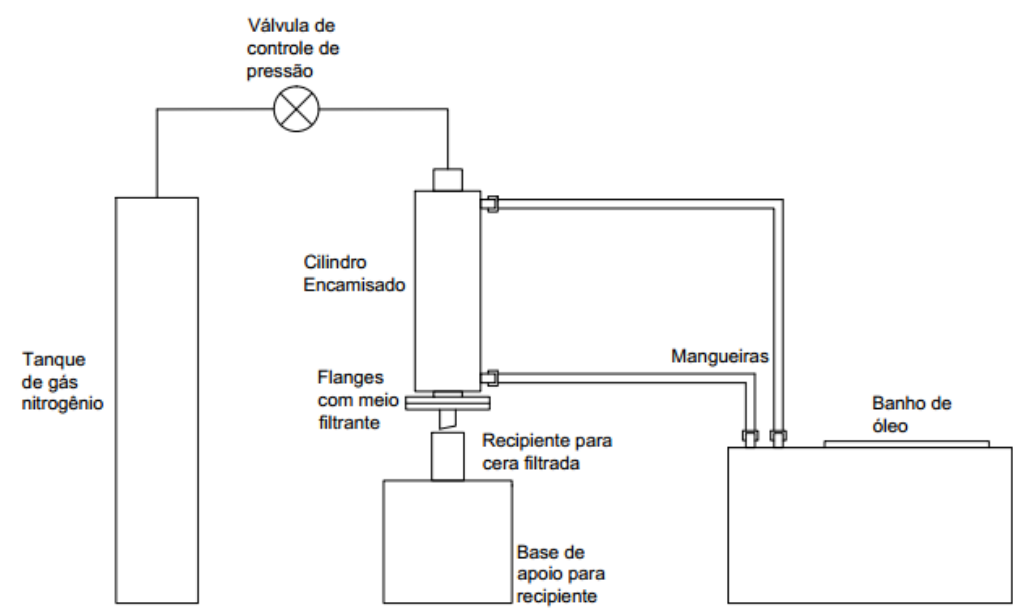

\subsection{Teor de inorgânicos}

O teor de inorgânicos foi determinado a partir de $5 \mathrm{~g}$ de cera bruta e filtrada, em $500{ }^{\circ} \mathrm{C}$ por $2 \mathrm{~h}$ utilizando um forno mufla, fabricante Ind. Magnus, modelo 1109 (Nazato et al. 2012).

\section{RESULTADOS E DISCUSSÃO}

\subsection{Delineamento Composto Central Rotacional}

Na Tabela 2 são observados os níveis das variáveis reais e codificadas, assim como a resposta avaliada a massa de cera filtrada. O delineamento composto central rotacional foi gerado através do software STATISTICA Versão 10.0 da Statsoft Inc. (2010).

Tabela 2 - Ensaios de filtração.

\begin{tabular}{|c|c|c|c|}
\hline Ensaios & Temperatura $\left({ }^{\circ} \mathrm{C}\right)$ & Pressão (bar) & Massa filtrada $(\mathrm{g})$ \\
\hline 1 & $144,36(-1)$ & $1,44(-1)$ & 5,39 \\
\hline 2 & $165,64(1)$ & $1,44(-1)$ & 8,50 \\
\hline 3 & $144,36(-1)$ & $3,56(1)$ & 8,94 \\
\hline 4 & $165,64(1)$ & $3,56(1)$ & 8,85 \\
\hline 5 & $140(-1,41)$ & $2,5(0)$ & 6,89 \\
\hline 6 & $170(1,41)$ & $2,5(0)$ & 13,71 \\
\hline 7 & $155(0)$ & $1(-1,41)$ & 5,85 \\
\hline
\end{tabular}




\begin{tabular}{|c|c|c|c|}
\hline 8 & $155(0)$ & $4(1,41)$ & 8,42 \\
\hline 9 & $155(0)$ & $2,5(0)$ & 5,69 \\
\hline 10 & $155(0)$ & $2,5(0)$ & 4,60 \\
\hline 11 & $155(0)$ & $2,5(0)$ & 5,25 \\
\hline
\end{tabular}

Analisando os resultados para o rendimento de massa de cera filtrada, verificou-se que o melhor rendimento atingido foi com a temperatura de $170{ }^{\circ} \mathrm{C}$ e pressão de 2,5 bar. $\mathrm{Na}$ Tabela 3 está apresentada os parâmetros da ANOVA. Observou-se que o $\mathrm{F}_{\text {calc }}$ é cerca de 2,5 vezes maior que o $\mathrm{F}_{\text {tab. }}$. Com isso, o modelo proposto foi preditivo e a porcentagem de variação $\left(\mathrm{R}^{2}\right)$ explicada pelo modelo foi adequada $\left(\mathrm{R}^{2}=89,17 \%\right)$. Sendo assim, pode-se concluir que o modelo se ajustou bem aos dados experimentais.

A Figura 2a apresenta o diagrama de Pareto. Pode-se ver graficamente os parâmetros que foram mais significativos na operação de filtração da cera. A Figura $2 b$ apresenta a curva de contorno em função da pressão e temperatura para a massa de cera filtrada.

Baseado nas imagens da Figura 2, a temperatura foi o parâmetro que apresentou maior significância. $\mathrm{O}$ melhor valor de temperatura se concentra em $170{ }^{\circ} \mathrm{C}$. Isto pode ser comprovado pelo fato de que quanto maior a temperatura, menor será a viscosidade da cera (Irving H. Shames 2002). Sendo assim, mais facilmente a cera escoa pelo meio filtrante.

Em relação à pressão, este parâmetro não foi tão significativo quanto à temperatura. Estudos mostraram que o mesmo meio filtrante pode apresentar diferenças em termos da taxa de filtração e clarificação do filtrado (Koch \& Krammer 2015). Pode ocorrer de as partículas sólidas bloquearam parcialmente o meio filtrante logo após o início do teste de filtração. Isso acaba sendo relatado na literatura como uma natureza estatística, mesmo em filtros perfeitamente homogêneos. Desta forma, a pressão tem uma influência de maior significância nos estágios inicias da filtração, antes da formação completa da torta (Roussel et al. 2007). Baseado nestes estudos, e nas imagens da Figura 2, bem como nos resultados apresentados na Tabela 2, a pressão escolhida foi de 2,5 bar. Além de cumprir com o seu objetivo, forçar o escoamento da cera através do meio filtrante, também considera o lado econômico, pois com menos pressão necessita-se menos gás nitrogênio.

Tabela 2 - Anova para a massa de cera filtrada.

\begin{tabular}{|c|c|c|c|c|c|}
\hline Fonte & SQ & GL & MQ & F calc & F tab \\
\hline Regressão & 64,53 & 5 & 12,91 & \multirow{2}{*}{8,69} & \multirow{2}{*}{$\mathrm{F}_{0,90,5,5}=3,45$} \\
\hline Resíduo & 7,43 & 5 & 1,49 & & \\
\cline { 1 - 4 } Falta de ajuste & 6,83 & 3 & 2,28 & & \\
\hline Erro puro & 0,60 & 2 & 0,30 & & \\
\hline Total & 68,61 & 10 & & & \\
\hline
\end{tabular}


Figura 2 - a) diagrama de Pareto e b) curvas de contorno em função da pressão e temperatura para a massa de cera filtrada.
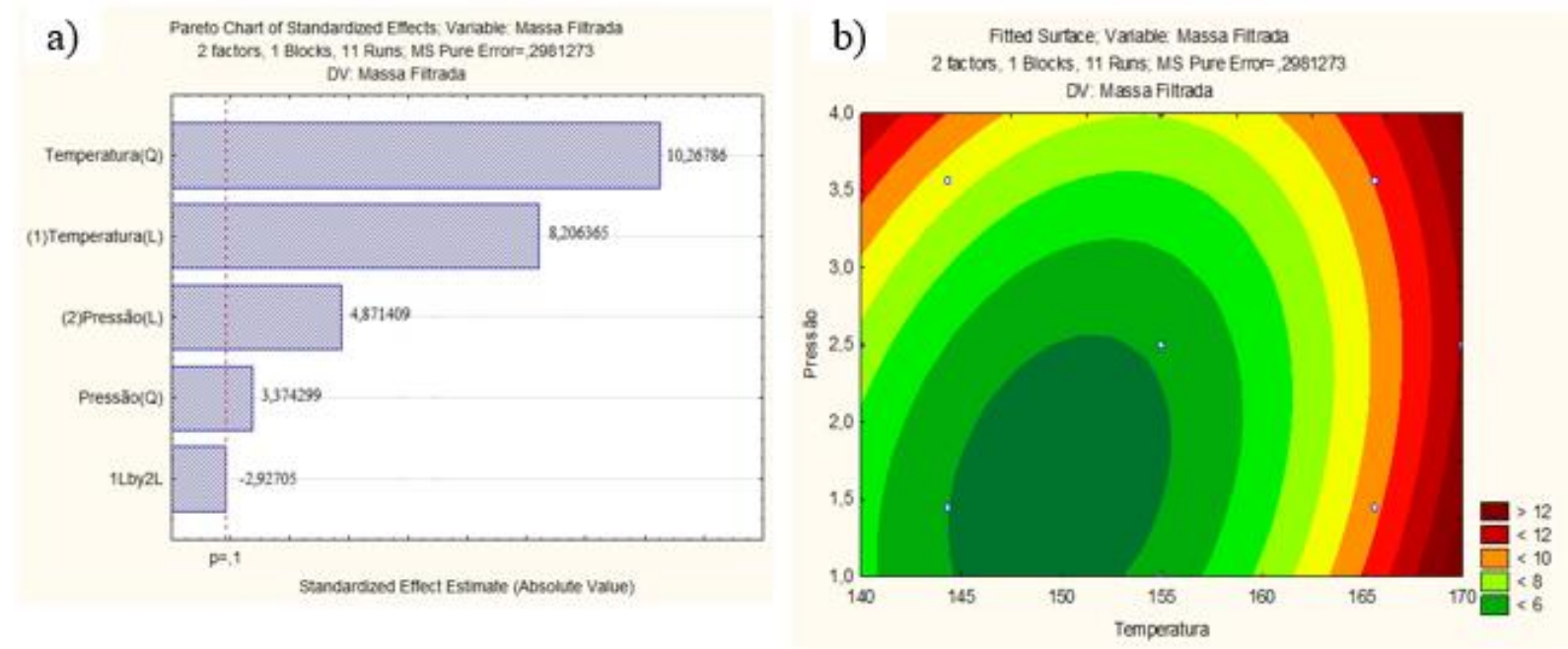

\subsection{Teor de inorgânicos}

$\mathrm{Na}$ Tabela 3 estão apresentados os valores das porcentagens de remoção de inorgânicos para cada ensaio de filtração.

Tabela 3 - Valores da porcentagem de remoção de inorgânicos.

\begin{tabular}{|c|c|c|c|c|c|c|c|c|c|c|c|}
\hline Ensaios & 1 & 2 & 3 & 4 & 5 & 6 & 7 & 8 & 9 & 10 & 11 \\
\hline $\begin{array}{c}\text { Porcentagem } \\
\text { de remoção de } \\
\text { sólidos }\end{array}$ & 98,89 & 99,74 & 99,83 & 99,84 & 99,72 & 99,74 & 98,32 & 99,81 & 99,74 & 99,76 & 99,83 \\
\hline
\end{tabular}

Observa-se que, em todos os ensaios, o teor de remoção de inorgânicos foi superior a 98\%. Logo, o meio filtrante aplicado foi capaz de reter grande parte das impurezas garantindo uma cera filtrada de ótima qualidade.

\section{CONCLUSÃO}

No presente trabalho efetuou-se a filtração de cera bruta obtida por pirólise de resíduos plásticos. Utilizou-se um meio filtrante sem tratamento físico. Para melhorar a eficiência de filtração, a perlita foi utilizada como auxiliar de filtração. Os resultados obtidos através do planejamento experimental, modelo DCCR, foram satisfatórios. O melhor resultado de massa filtrada foi de $13,71 \mathrm{~g}$, obtida com a temperatura de $170{ }^{\circ} \mathrm{C}$ e pressão de 2,5 bar. Estes valores de temperatura e pressão compõe a melhor condição operacional. O percentual médio de remoção das impurezas foi de, aproximadamente, 99,6\%. O produto obteve um elevado grau de pureza. 


\section{REFERÊNCIAS}

$\mathrm{Du}, \mathrm{L}$. et al., 2011. A study on enhancement of filtration process with filter aids diatomaceous earth and wood pulp cellulose. Chinese Journal of Chemical Engineering, 19(5), pp.792-798. Available at: http://dx.doi.org/10.1016/S1004-9541(11)60058-X.

Irving H. Shames, 2002. Mechanics of Fluids (Mcgraw-Hill Series in Mechanical Engineering) 4th edition, Mcgraw-Hill (Tx).

Koch, M. \& Krammer, G., 2015. Permeability distribution for filter media characterization in solid liquid filtration. Powder Technology, 271, pp.182-186. Available at: http://dx.doi.org/10.1016/j.powtec.2014.10.042.

Miandad, R. et al., 2016. Influence of temperature and reaction time on the conversion of polystyrene waste to pyrolysis liquid oil. Waste Management, 58, pp.250-259. Available at: http://dx.doi.org/10.1016/j.wasman.2016.09.023.

Mullin, W.J., 2001. Crystallization 4th edition, Butterworth-Heinemann.

Nazato, C. et al., 2012. CERA DE CANA-DE-AÇUCAR (Saccharum officinarum L.) NA FORMULAÇÃO DE UM BRILHO LABIAL A PARTIR DE EXTRAÇÃO SIMPLES E POR BIOETANOL. Revista Brasileira de Produtos Agroindustriais, 14(4), pp.389-396.

Purchas, D.B. \& Sutherland, K., 2002. Handbook of Filter Media 2nd edition, Elsevier Science.

Rodrigues, M.I. \& Iemma, A.F., 2014. Planejamento de Experimentos e Otimização de Processos $3^{\mathrm{a}}$ edição., Cárita Editora.

Roussel, N., Nguyen, T.L.H. \& Coussot, P., 2007. General Probabilistic Approach to the Filtration Process. Physical Review Letters, 98(11), p.114502 (1-4).

Wiley-VCH, 2011. Ullmann's Encyclopedia of Industrial Chemistry 1st edition, JOHN WILEY REFERENCE. 